\title{
The Relationship between Neuroticism and Experience of Workplace Ostracism in New Employees
}

\author{
Xiang Zhang, Liangtie Dai \\ School of Management, Jinan University, Guangzhou, China \\ Email: zhangxiang0207@126.com
}

Received 9 January 2015; accepted 24 January 2015; published 29 January 2015

Copyright (C) 2015 by authors and Scientific Research Publishing Inc.

This work is licensed under the Creative Commons Attribution International License (CC BY). http://creativecommons.org/licenses/by/4.0/

c) (i) Open Access

\begin{abstract}
The experience of workplace ostracism describes the extent to which an individual perceives that he or she is ignored or excluded at work. This article explores the intermediary and regulatory role of interpersonal trust and task interdependence, in order to identify how neuroticism affects new employees' experience of workplace ostracism. By using the data from 410 new enterprise staffs, the result shows that neuroticism positively affects the experience of workplace ostracism, and that interpersonal trust plays an intermediate role. The lower the task interdependence scores are, the stronger the relationship between neuroticism and experience of workplace ostracism will be.
\end{abstract}

\section{Keywords}

Neuroticism, Workplace Ostracism, Interpersonal Trust, Task Interdependence

\section{Introduction}

Ostracism is a universal social phenomenon existing in the real life of human society, which has drawn great attention by psychologists and sociologists. It affects the way people treat and are treated by others to a great extent [1]. In the past 20 years, ostracism within enterprises starts being studied widely and thoroughly. Wang and Liu (2013) proposed that workplace is one of the social situations in which ostracism occurs frequently. It has great value in theory and practical significance to explore workplace ostracism in China [2].

Continuous acquisition of new strength hallows organizations grow sustainably and develop innovatively. Due to the drastic competition and complicate workplace relations, the phenomenon that old employees reject new employees is common. The old staffs may frown upon the new employees with pushy personality, and worry about the threat on their own status. Then the old staffs may ignore the new staffs' existence, or suppress 
their development deliberately. New employees are not familiar with the work content, enterprise culture and interpersonal relationships, so it is unavoidable to experience an adaptive phase. The higher the proportion of new employee is, the more organizational tolerance and resilience of employees is demanded. In the process, the conflict between older employees and new employees is inevitable. Because almost all modern enterprise calls for a harmonious interpersonal atmosphere, the contradiction between the new and older workers tends to take more indirect form. Ostracism is a typical concealed form of conflict. The poisonous relationship can eventually bring about internecine, and have negative impact on enterprise's vitality and cohesion. Therefore, how to reduce or prevent older workers from rejecting new employees to realize the healthy development of working relationship, is now an issue that needed to be addressed as a matter of urgency.

Existing researches have been widely explored the consequences and mechanism of workplace ostracism. In addition to taking appropriate measures to reduce the negative effects of ostracism, the more effective action is to avoid the happening of these deviant behaviors. Therefore, it is necessary to explore how workplace ostracism occurs, that is to say, we must explore what factors can lead workplace ostracism, and how these factors affect workplace ostracism. Ferries (2008) initially puts forward the concept of workplace ostracism, which is defined as the extent to which an individual perceives that he or she is ignored or excluded at work [3]. By definition, it is obvious that workplace ostracism is a kind of subjective experiences. Others in the organization may be mistaken for acting rejecting behavior intentionally, instead, others mean to reject someone may not be regarded as excluders. The standard to judge whether a behavior is ostracism depends on individual subjective and evaluation [2]. Individual characteristic is a core factor affecting subjective cognition, so we suggest that neuroticism is one of personality traits that contribute to workplace ostracism. This paper is aim to explore the relationship between neuroticism and experience of workplace ostracism, to help managers better understanding the present situation of work relationship. It offers a new principle and direction for creating a harmonious working environment.

\section{Theoretical Background and Hypotheses}

\subsection{The Direct Impact of Neuroticism}

Victim precipitation theory proposes that individuals don't select targets at random to act deviant behaviors. The targets play an important role in their own mistreatment [4]. Olweus's (1978) research revealed that provocative individuals (e.g., aggressive, hostile, disagreeable) were more likely to be bullied by others [5]. Similarly, this phenomenon also occurs in workplace. Tepper (2009) found that workers characterized as being emotionally unstable (i.e., neuroticism) were likely to be treated abusively by their supervisors [6]. On the basis of these findings, we argue that staffs that have negative characteristics or display negative behaviors at work are more likely to become the target of mistreatment. Highly neurotic individuals easily appear to be impulsive, hostile or depressive. Due to these characteristics which are undesirable in social life, the person with high neuroticism is more likely to be regarded as the target of deviant behaviors. In one case, highly neurotic individuals are more sensitive so that they perceive the motives behind conduct keenly. In another scenario, under the influence of instable emotion, highly neurotic individuals may mistakenly assess others' actions or intentions. In this article, we expect that neuroticism will be associated with higher levels of workplace ostracism. Thus, we predict that neuroticism has significant direct effect on experience of workplace ostracism.

Hypothesis 1: neuroticism has significant direct effect on experience of workplace ostracism.

\subsection{The Mediating Role of Interpersonal Trust}

According to social exchange theory, positive interaction between people is built on the norm of reciprocity. Thus, why negative interaction (i.e., workplace ostracism) happens can be explained by the specific relationship between both sides to some extent. As mentioned, highly neurotic individuals are so sensitive that they are often suspicious about others' motives, so it is hard for them to view others as trustworthy [4]. Individuals in the same group are generally expected to work cooperatively to achieve common organizational goals [7]. Mutual suspicion violates this expectation and trust between coworkers. Once interpersonal trust is damaged, individuals are often unwilling to interact with those whom are distrusted [8], and reluctant to maintain these relationships with those who have violated their trust [9]. Considered together, neuroticism and the interpersonal trust are generally viewed as a factor inflecting experience of workplace ostracism. Thus, we predict that interpersonal trust me- 
diates the positive relationship between neuroticism and experience of workplace ostracism.

Hypothesis 2: interpersonal trust mediates the positive relationship between neuroticism and experience of workplace ostracism.

\subsection{Moderating Role of Task Interdependence}

However, not all employees who are highly neurotic will experience the same level of workplace ostracism. Team characteristics also likely influence employees' subjective cognition about whether or not to be excluded by others. A prominent characteristic of teams that relates to the interaction between group members is task inter dependence. Task interdependence was defined by Campion (1993) as the level in which group members interact and depend on one another to accomplish the work [10]. Individuals' deviant behaviors to others will cause a greater loss to themselves under high task interdependence setting, thus they believe that the coworkers will not violate the relationship intentionally. We offer the following prediction:

Hypothesis 3: The strength of the effect of neuroticism on experience of workplace ostracism is influenced by task interdependence, such that the relationship will be stronger when the task interdependence is low and will be weaker when the task interdependence is high.

The whole conceptual model of this study is illustrated in Figure 1.

\section{Method}

\subsection{Sample}

The participants were 410 new employees from varied cities including Guangzhou, Changsha and Nanchang. First, $53 \%$ of the respondents were women, and $47 \%$ were men. In the sample, one-third of the respondents were under 25. Half of the respondents were between 25 and 30, and 17\% were over 30. Finally, Eighteen percent were high school graduates. $14 \%$ had completed a professional diploma program. $39 \%$ had attended college education, and $29 \%$ had finished a master's degree.

\subsection{Measures}

All measures used in this study have been previously validated in published research and all measures adopted a 5-point Likert scale ( 1 = strongly disagree to $5=$ strongly agree).

Neuroticism was measured using the Chinese Big Five Personality Inventory brief version (CBF-PI-B), which is developed on the basis of Chinese Big Five Personality Inventory (CBF-PI-B) by Wang (2011). According to the research purposes, we chose items related to neuroticism. It consists of 8 items. Cronbach's $\alpha$ for neuroticism is 0.81 . Sample items included: "I often worry about whether bad things may happen." [11]-[13].

Workplace ostracism was investigated through the workplace ostracism scale (WOS) developed by Ferris et al. (2008). It is a self-report questionnaire composed of 10 items. Ferris tested workplace ostracism to be a unidimensional factor structure. Using data from 4 separate samples, the Cronbach's $\alpha$ of WOS is range from0.89 to 0.96 . Sample items included: "Others ignored you at work", "You involuntarily sat alone in a crowded lunchroom at work" and "Others at work shut you out of the conversation." [2].

For interpersonal trust, we utilized the 11-item measure of interpersonal trust developed by McAllister (1995). This measure includes 5 items for affect-based trust and 6 items for cognition-based trust. A sample item for affect-based trust is "We have a sharing relationship. We can both freely share our ideas, feelings, and hopes." A

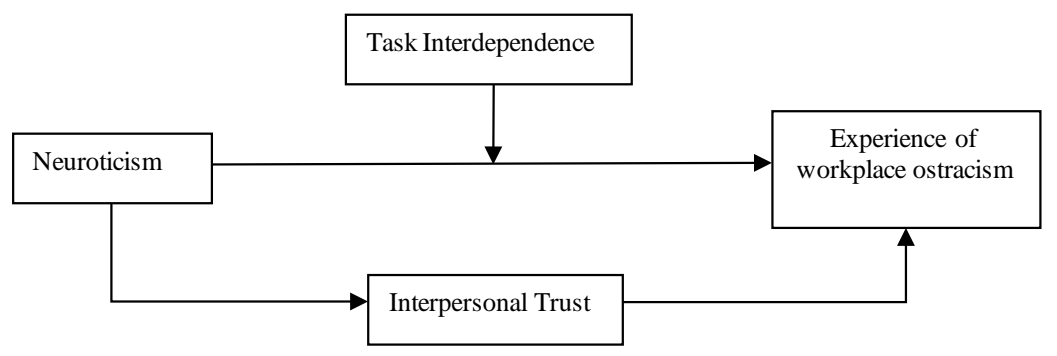

Figure 1. The conceptual model of the study. 
sample item for cognition-based trust is "This person approaches his/her job with professionalism and dedication”. Cronbach's alphas for the affect- and cognition-based trust measures are 0.89 and 0.91 , respectively [14].

New employees reported task interdependence by answering 5 questions developed by Pearce and Gregersen (1991). A sample item is "I work closely with others in doing my work". The alpha reliability coefficient was $0.76[15]$.

\subsection{Procedure}

We contacted one new employee representative for each participating organization by e-mail. Their duty was to distribute the questionnaire to potential participants. The survey items were used to assess the participants' level of neuroticism, experience of workplace ostracism, interpersonal trust and task interdependence. The questionnaire was collected and analyzed uniformly. According to the purpose of this research, we tested the level of common method bias firstly, as well as reliability and validity of each questionnaire. Then, Pearson's correlation was applied to initially test the possible relationship between these variables (see Table 1). Finally, for verifying the role of mediator (interpersonal trust) and moderator (task interdependence) in the relationship between independent variable (neuroticism) and dependent variable (experience of workplace ostracism), we have used linear regression (see Table 2 and Table 3).

\section{Results}

\subsection{Common Method Bias Test}

Considering that all the items in the questionnaire were filled out by the same participant, we must test the

Table 1. Pearson's correlations.

\begin{tabular}{|c|c|c|c|c|c|c|}
\hline & $\mathbf{M}$ & SD & 1 & 2 & 3 & 4 \\
\hline 1. Neuroticism & 2.37 & 0.726 & 1 & & & \\
\hline 2. Interpersonal Trust & 3.45 & 0.815 & $-0.573^{* *}$ & 1 & & \\
\hline 3. Task Interdependence & 3.15 & 0.652 & $0.367^{* *}$ & 0.087 & 1 & \\
\hline 4. Workplace Ostracism & 2.57 & 0.813 & $0.571^{* *}$ & $-0.703^{* *}$ & $-0.173^{* *}$ & 1 \\
\hline
\end{tabular}

${ }^{* *} \mathrm{p}<0.01$.

Table 2. Relationship between neuroticism and workplace ostracism: the mediating effect of interpersonal trust.

\begin{tabular}{|c|c|c|c|}
\hline \multirow{2}{*}{ Dependent Variable } & \multicolumn{2}{|c|}{ Workplace Ostracism } & \multirow{2}{*}{ Interpersonal Trust } \\
\hline & Model 1 & Model 2 & \\
\hline \multicolumn{4}{|l|}{ Contorl Variable } \\
\hline Gender & -0.032 & -0.067 & -0.066 \\
\hline Age & -0.183 & 0.116 & 0.127 \\
\hline Education & -0.099 & -0.062 & 0.070 \\
\hline \multicolumn{4}{|l|}{ Independent Variable } \\
\hline Neuroticism & $0.535^{* * *}$ & $0.241^{* * *}$ & $-0.555^{* * *}$ \\
\hline \multicolumn{4}{|l|}{ Mediator } \\
\hline Interpersonal Trust & & $-0.529^{* * *}$ & \\
\hline $\mathbf{R}^{2}$ & 0.398 & 0.578 & 0.358 \\
\hline Adjusted $\mathbf{R}^{2}$ & 0.387 & 0.569 & 0.347 \\
\hline $\mathbf{F}$ & $37.923^{* * *}$ & $68.561^{* * *}$ & $32.007^{* * *}$ \\
\hline$\Delta \mathbf{R}^{2}$ & 0.270 & 0.180 & 0.290 \\
\hline
\end{tabular}

\footnotetext{
${ }^{* * *} \mathrm{p}<0.001$.
} 
Table 3. Relationship between neuroticism and workplace ostracism: the moderating effect of task interdependence.

\begin{tabular}{|c|c|c|c|}
\hline \multirow{2}{*}{ Dependent Variable } & \multicolumn{3}{|c|}{ Workplace Ostracism } \\
\hline & Model 1 & Model 2 & Model 3 \\
\hline \multicolumn{4}{|l|}{ Contorl Variable } \\
\hline Gender & -0.032 & -0.032 & -0.017 \\
\hline Age & -0.183 & -0.147 & -0.141 \\
\hline Education & 0.099 & -0.089 & -0.091 \\
\hline \multicolumn{4}{|l|}{ Independent Variable } \\
\hline Neuroticism & $0.535^{* * *}$ & $0.694^{* * *}$ & $0.715^{* * *}$ \\
\hline \multicolumn{4}{|l|}{ Moderator } \\
\hline Task Interdependence & & $-0.418^{* * *}$ & $-0.463^{* * *}$ \\
\hline \multicolumn{4}{|l|}{ Interaction } \\
\hline Neuroticism $\times$ Task Interdependence & & & $-0.134^{* * *}$ \\
\hline $\mathbf{R}^{2}$ & 0.398 & 0.546 & 0.562 \\
\hline Adjusted $\mathbf{R}^{2}$ & 0.387 & 0.537 & 0.552 \\
\hline $\mathbf{F}$ & $37.923^{* * *}$ & $60.352^{* * *}$ & $57.081^{* * *}$ \\
\hline$\triangle \mathbf{R}^{2}$ & 0.270 & 0.148 & 0.016 \\
\hline
\end{tabular}

${ }^{* * *} \mathrm{p}<0.001$.

common method bias to ensure the data scientific. According to the single factor test proposed by Harman, the result shows that single factor can only explained $28 \%$ of the total variance, so there is no common method variance problem.

\subsection{Correlation Analysis}

The means, standard variances and Pearson's correlation coefficients have been shown in Table 1. Neuroticism and interpersonal trust seem to be inversely related $(r=-0.573, p<0.01)$. Neuroticism is positively related to workplace ostracism $(\mathrm{r}=0.571, \mathrm{p}<0.01)$. The means of interpersonal trust and task interdependence are relative higher ( $M=3.45$ and 3.15). On one hand, we can infer that the organizations have nurtured high level of trust between the old staffs and new employees, meanwhile, the interdependence on each other at work has been deepen. On the other hand, the participants may be affected by social desirability distortion, thus they responded as others expected. The reliability and validity of all measures are comparatively ideal (Cronbach’s $\alpha>0.8$ ).

\subsection{Mediating Effect Analysis}

In order to prove the mediating effect, we must meet the following requirements: 1) Independent variable has significant effect on dependent variable; 2) Independent variable has significant effect on intermediary variable; 3) Intermediary variable has significant effect on dependent variable; 4) After putting independent variable and intermediary variable into equations simultaneously, if the mediator becomes significant without significant independent variable, the mediator is a full intermediate variable. If both mediator and independent variable are significant, the mediator plays a role of partly intermediate variable.

As shown in Table 2, neuroticism could significantly positive predict the experience of workplace ostracism ( $\mathrm{r}=0.535$, $\mathrm{p}<0.001)$. The higher the neuroticism, the stronger the experience of workplace ostracism in Chinese enterprises is. The conclusion is consistent with the view of Wu (2011) [16]. Employees with high neuroticism always stay over sensitive to social behaviors and cues. They tend to make negative comments about others’ behaviors. Controlling demographic variables (e.g., gender, age, and education), neuroticism could significantly negative predict the interpersonal trust $(r=-0.555, \mathrm{p}<0.001)$. Neuroticism could reduce the trust between employees. The conclusion is consistent with Scott (2013) [4]. Putting interpersonal trust into the equation, 
neuroticism still showed the significant predictable function of experience of workplace ostracism. It means that interpersonal trust plays a part mediating role in the influences of neuroticism to experience of workplace ostracism.

\subsection{Moderating Effect Analysis}

As shown in Table 3, the interaction (neuroticism $\times$ task interdependence) has significantly negative impact on experience of workplace ostracism $(\mathrm{r}=-0.134, \mathrm{p}<0.001)$. It indicated that the lower the task interdependence scores, the stronger the relationship between neuroticism and workplace ostracism will be.

\section{Discussion}

There are several researches that highlighted some antecedents of workplace ostracism [2] [4], yet little has been verified by the data in Chinese organizations. We developed and tested a model to realize why certain employees are more likely to get unpleasant sensory and emotional experience of ostracism. The results provide support for our assumptions.

The results of correlation analysis indicated that there was a significant positive correlation between neuroticism and experience of workplace ostracism in new employees. Employees high in neuroticism are more likely to experience workplace ostracism, so hypothesis 1 had been tested. Facing the change of work settings and job roles, new staffs with high neuroticism are more prone to negative emotions. As a result, it would be particularly important for managers to have an accurate understanding of new employees' personalities. Enterprise managers must provide timely intervention (i.e., provide training, counseling, and social support). Thus the negative feelings of new staffs can be lower and the ostracism behaviors to them can also be reduced.

Hierarchical regression analysis showed that interpersonal trust played a partial mediating role between neuroticism and experience of workplace ostracism, thus hypothesis 2 had been tested. With higher interpersonal trust, the individual would prefer to be frank and honest with each other. Then, they get to know each other better and evaluate others' actions positively. As a new member of the organization, the new employee is eager to get approval from the old staffs whose behavioral feedback is the most direct source of experience. To establishing harmonious relationship between the employees, managers should pay close attention to the new employees' psychological dynamic, create good atmosphere and carry out team activities or other approaches for interpersonal interaction to maintain the steady trust relationship of coworkers.

In addition, we found that task interdependence can effectively weaken the relationship between neuroticism and experience of workplace ostracism. When task interdependence is relatively high, experience of workplace ostracism increased as the level of neuroticism increased. Similarly, When task interdependence is relatively low, experience of workplace ostracism had also increased, but it increased faster than that under high task interdependence. Robinson (2013) proposed that team task interdependence was an important factor of workplace ostracism [17], which had been tested as well as the hypothesis 3 in this research. Task interdependence can directly affect the quantity and quality of interpersonal interactions. High task interdependence means that more social connections can be accumulated which help making employees familiar with each other and evaluate actions objectively. Besides, when someone's work is closely linked to others' work, acting negative behavior will cause more psychic costs. So it is effective to inhibit ostracism behaviors. Thus, the managers should create the culture atmosphere of cooperation through designing job scientifically to reduce the likelihood of workplace ostracism.

\section{Limitations and Directions for Future Research}

This research built and empirically tested a model that links employee personality traits (i.e., neuroticism) with experience of workplace ostracism. In addition, we also spotlighted the relationship between group members and the team characteristic which are largely ignored in the past. We demonstrated the framework for understanding why and how employees experience workplace ostracism. Meanwhile, we confirmed the mediating role of interpersonal trust and the moderating role of task interdependence. Our research explores several antecedents from diverse perspectives, including individual characteristics, relational factors, and team characteristics that are potentially related to workplace ostracism. These results contribute much theoretic and practical enlightenment. However, there are still many deficiencies that need further improvement. 
First, workplace ostracism is a kind of covert negative behaviors that is difficult to observe directly. The construct of workplace ostracism measured in this study is exactly the subjective feelings of staffs. Because of the ambiguous behavioral intention, we can't rule out the possibility that the participants may exaggerate or deny the extent of ostracism. Future study can try to collect data from various sources (i.e., excluder, victim). It is feasible to investigate the field in a laboratory to control relative variables.

Second, the main method adopted in this research was cross-sectional design, so the change over time was not considered. The new employees are at a crucial stage of career development, and will face many important decisions about the future. As the interaction with old staffs get more and more frequent, the new employees' attitude and perception may also change over time. Thus, it is worthy of further researching to design a longitudinal or experimental study to tease out the causality of workplace ostracism, particularly with regard to the experience of the new staffs over time.

Third, interpersonal trust is playing a part mediating role between neuroticism and experience of workplace ostracism. That is to say, there may be other intermediary variables. Besides, task interdependence plays an important role indeed in the conceptual model of this study, but other moderators may also exist which can affect the path coefficients. This research explored antecedents of workplace ostracism from multiple perspectives, but the selected variables were limited. Further research should construct an integrated model to take into account other factors from inside or outside the organization, as well as organizational, team and individual levels.

\section{Conclusion}

In this paper, we call attention to the antecedents and formation mechanism of experience of workplace ostracism. Through empirical research, we have developed a model of workplace ostracism that shows how individual characteristics, work-related factors, and team characteristics impact on the experience of workplace ostracism. This article helps us to better understand the form of the new employees' experience of workplace ostracism, and may provide significant hints for the staff relation management in the future. We believe that the conception and the result can provide reference for further study as well as to provide guidance for the practical management of organizational behavior.

\section{References}

[1] Williams, K.D. (2002) Ostracism: The Power of Silence. 1st Edition, Guilford Press, New York.

[2] Wang, H.Y. and Liu, Y.F. (2013) A Review of Frontier Research on Workplace Ostracism and Future Prospects. Foreign Economics and Management, 35, 31-39.

[3] Ferris, D.L., Brown, D.J., Berry, J.W. and Lian, H. (2008) The Development and Validation of the Workplace Ostracism Scale. Journal of Applied Psychology, 93, 1348-1366. http://dx.doi.org/10.1037/a0012743

[4] Scott, K.L., Restubog, S.L.D. and Zagenczyk, T.J. (2013) A Social Exchange-Based Model of the Antecedents of Workplace Exclusion. Journal of Applied Psychology, 98, 37-48. http://dx.doi.org/10.1037/a0030135

[5] Olweus, D. (1978) Aggression in Schools: Bullies and Whipping Boys. Hemisphere, Washington DC.

[6] Tepper, B.J., Carr, J.C., Breaux, D.M., Geider, S., Hu, C. and Hua, W. (2009) Abusive Supervision, Intentions to Quit, and Employees' Workplace Deviance: A Power/Dependence Analysis. Organizational Behavior and Human Decision Processes, 109, 156-167. http://dx.doi.org/10.1016/j.obhdp.2009.03.004

[7] Dunn, J.R. and Schweitzer, M.E. (2004) Invidious Comparisons and Insidious Behaviors: Envy and Social Undermining at Work. Working Paper. http://dx.doi.org/10.1111/j.1744-6570.1993.tb01571.x

[8] Kramer, R.M. (1999) Trust and Distrust in Organizations: Emerging Perspectives, Enduring Questions. Annual Review of Psychology, 50, 69-598. http://dx.doi.org/10.1146/annurev.psych.50.1.569

[9] Robinson, S.L. (1996) Trust and Breach of the Psychological Contract. Administrative Science Quarterly, 41, 574-599. http://dx.doi.org/10.2307/2393868

[10] Campion, M.A., Medsker, G.J. and Higgs, C.A. (1993) Relations between Workgroup Characteristics and Effectiveness: Implications for Designing Effective Work Groups. Personnel Psychology, 46, 823-850. http://dx.doi.org/10.1111/j.1744-6570.1993.tb01571.X

[11] Wang, M.C., Dai, X.Y. and Yao, S.Q. (2010) Development of the Chinese Big Five Personality Inventory (CBF-PI) I: Theoretical Framework and Reliability Analysis. Chinese Journal of Clinical Psychology, 18, 545-548.

[12] Wang, M.C., Dai, X.Y. and Yao, S.Q. (2010) Development of the Chinese Big Five Personality Inventory (CBF-PI) II: Validity Analysis. Chinese Journal of Clinical Psychology, 18, 687-690. 
[13] Wang, M.C., Dai, X.Y. and Yao, S.Q. (2011) Development of the Chinese Big Five Personality Inventory (CBF-PI) III: Psychometric Properties of CBF-PI Brief Version. Chinese Journal of Clinical Psychology, 19, 454-457.

[14] McAllister, D.J. (1995) Affect- and Cognition-Based Trust as Foundations for Interpersonal Cooperation in Organizations. Academy of Management Journal, 38, 24-59. http://dx.doi.org/10.2307/256727

[15] Pearce, J.L. and Gregersen, H.B. (1991) Task Interdependence and Extra Role Behavior: A Test of the Mediating Effects of Felt Responsibility. Journal of Applied Psychology, 76, 838-844. http://dx.doi.org/10.1037/0021-9010.76.6.838

[16] Wu, L.Z., Wei, L.Q. and Hui, C. (2011) Dispositional Antecedents and Consequences of Workplace Ostracism: An Empirical Examination. Frontiers of Business Research in China, 5, 23-44. http://dx.doi.org/10.1007/s11782-011-0119-2

[17] Robinsin, S.L., O’Reilly, J. and Wang, W. (2013) Invisible at Work: An Integrated Model of Workplace Ostracism. Journal of Management, 39, 203-231. http://dx.doi.org/10.1177/0149206312466141 
Scientific Research Publishing (SCIRP) is one of the largest Open Access journal publishers. It is currently publishing more than 200 open access, online, peer-reviewed journals covering a wide range of academic disciplines. SCIRP serves the worldwide academic communities and contributes to the progress and application of science with its publication.

Other selected journals from SCIRP are listed as below. Submit your manuscript to us via either submit@scirp.org or Online Submission Portal.
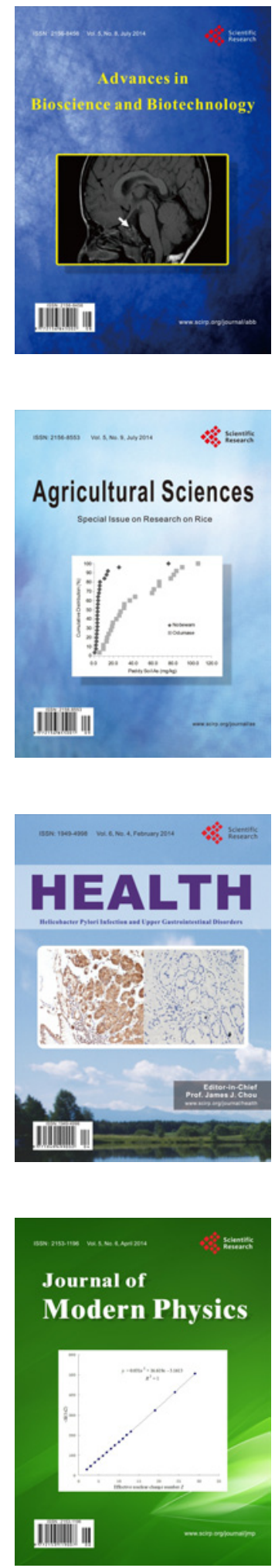
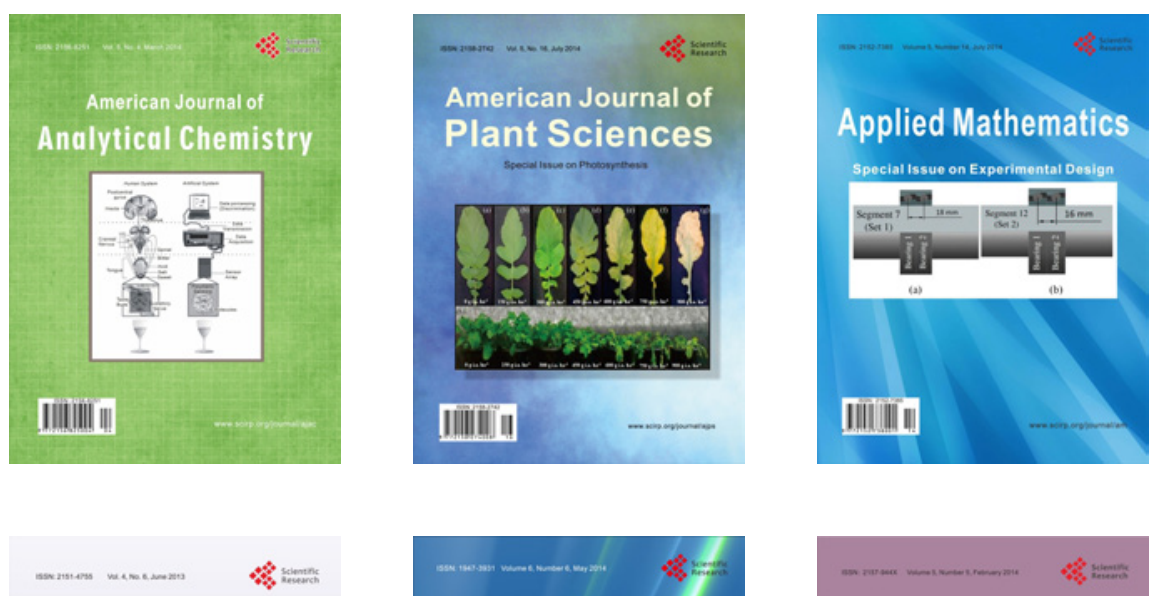

Creative Education
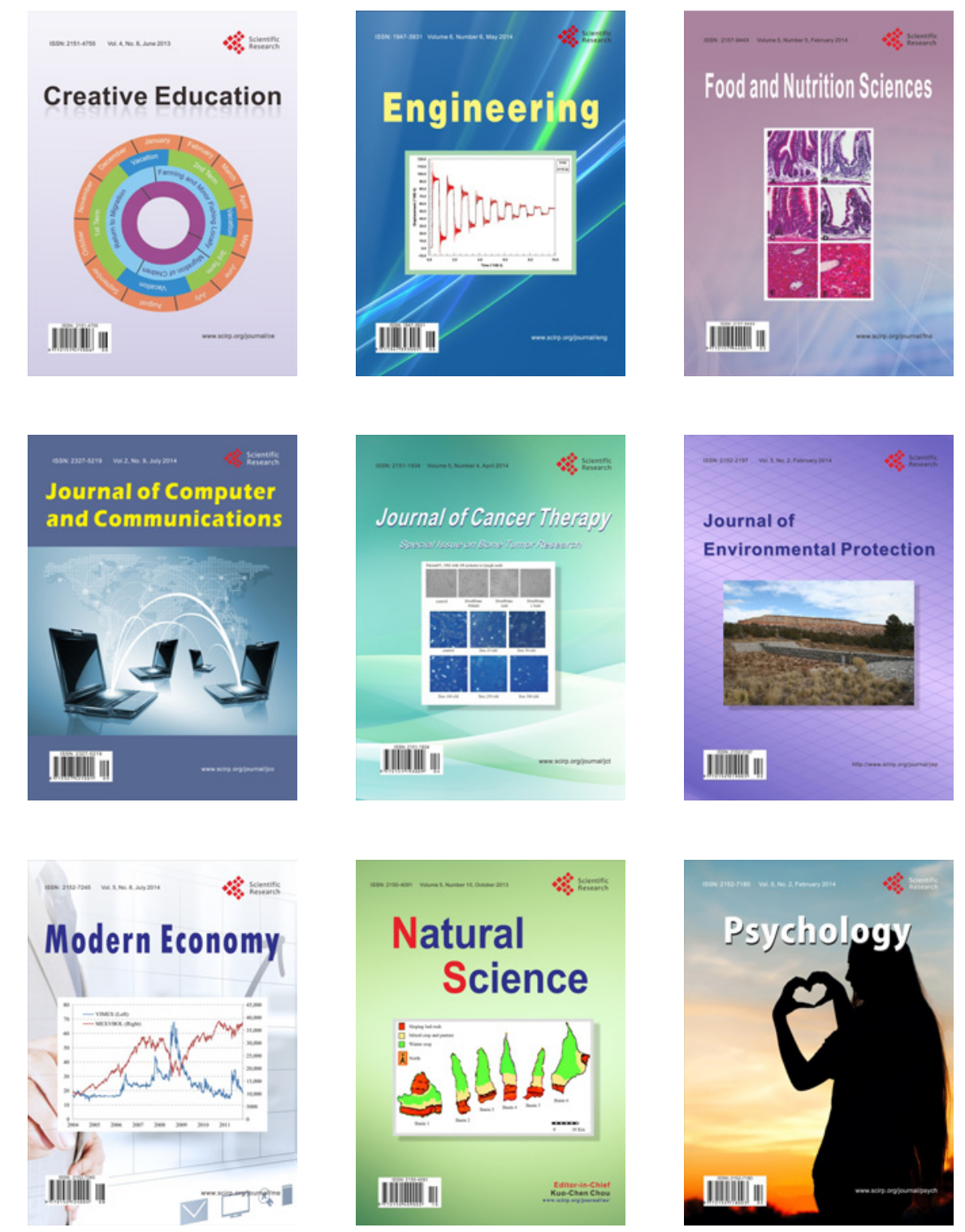